\title{
Indicadores de actividad científica en investigadores singulares: perfil bibliométrico de Eduardo Primo Yúfera, expresidente del CSIC
}

\author{
María Ayala-Gascón*, Rafael Aleixandre-Benavent**, \\ Asunción Gandía-Balaguer*
}

Resumen: Eduardo Primo Yúfera fue el fundador y director del Instituto de Agroquímica y Tecnología de los Alimentos hasta su nombramiento como presidente del Consejo Superior de Investigaciones Científicas. Es considerado el promotor e impulsor de la Ciencia y Tecnología de los Alimentos y de la Ecología Química en España, así como el precursor del modelo de sistema de innovación ( $\mathrm{I}+\mathrm{D}+\mathrm{i})$. A lo largo de su dilatada trayectoria investigadora, que duró 65 años, publicó 291 artículos científicos, con una media de 4,48 trabajos anuales. El 89,3\% los realizó en coautoría, colaborando con 161 autores diferentes. Su empeño por difundir la ciencia le llevó a crear en 1961 la Revista de Agroquímica y Tecnología de los Alimentos. El 50\% de su producción científica quedó recogida en esta revista aunque también publicó en más de 50 revistas científicas nacionales e internaciones, 36 de ellas incluidas en el Journal Citation Report, entre las que destacan por su mayor factor de impacto: Journal of Organic Chemistry, Planta y Journal of Agricultural and Food Chemistry, que se sitúan en el primer cuartil de sus áreas correspondientes. Los 86 artículos del autor que aparecen indexados en el SCI-Expanded han recibido un total 482 citas. Este estudio pone de relieve su actividad científica a través de indicadores bibliométricos de productividad, colaboración e impacto científico de sus publicaciones.

Palabras clave: Cienciometría, bibliometría, Eduardo Primo Yúfera, biografía, productividad científica, patrones de colaboración, impacto científico.

Scientific activity indicators of outstanding researchers: scientometric portrait of Eduardo Primo Yúfera, former president of CSIC (The Spanish National Research Council)

Abstract: Eduardo Primo Yúfera was the founder of the Institute of Agrochemicals and Food Technology (IATA-CSIC) and was its director until being appointed president of

* Instituto Universitario de Investigación «Doctor Viña Giner». Universidad Católica de Valencia San Vicente Mártir. Valencia. España. Correo-e: maria.ayala@ucv.es; asun.gandia@ucv.es.

** Unidad de Información e Investigación Social y Sanitaria-UISYS. Consejo Superior de Investigaciones Científicas-Universidad de Valencia. Valencia. España. Correo-e: rafael.aleixandre@uv.es.

Recibido: 13-09-2011; 2. ${ }^{a}$ versión: 25-01-2012; aceptado: 28-01-2012. 
the Spanish National Research Council. He is considered both patron and promoter of the fields of food science and technology and chemical ecology in Spain, as well as pioneer of the innovation system model $(R+D+i)$. Throughout his long research career, which lasted 65 years, he published 291 scientific papers, with an average of 4.48 papers per year. Of these, $89.3 \%$ were in collaboration with others: a total of 161 different coauthors. His efforts to disseminate science led him to create the Revista de Agroquimica $y$ Tecnología de los Alimentos in 1961. A total of 50\% of his scientific production was featured in this journal but his work was also published in more than 50 national and international scientific journals, of which 36 are included in the Journal Citation Report. Those with the highest impact factors are Journal of Organic Chemistry, Planta and Journal of Agricultural and Food Chemistry, which feature in the first quartile in their respective subject areas. The 86 articles listed in SCI-Expanded have received a total of 482 citations. This study highlights his scientific activity, using bibliometric indicators of productivity, collaboration and the scientific impact of his publications.

Keywords: Scientiometrics, bibliometrics, Eduardo Primo Yúfera, biography, scientific productivity, collaboration patterns, scientific impact.

Siglas: CEQA: Centro de Ecología Química Agrícola; CSIC: Consejo Superior de Investigaciones Científicas; IATA: Instituto de Agroquímica y Tecnología de Alimentos; ITQ: Instituto de Tecnología Química; UV: Universidad de Valencia; UPV: Universidad Politécnica de Valencia.

\section{Introducción}

Los estudios bio-bibliométricos se centran principalmente en el estudio biográfico de científicos e investigadores individuales junto con el análisis bibliométrico de sus publicaciones, correlacionando las publicaciones bibliográficas o académicas con los logros científicos de sus carreras (Mulla y otros, 2010). Algunos estudios bibliométricos han puesto de relieve la aportación a la ciencia de insignes científicos (Peters y Van Rann, 1999; Cardona, 2003; Kalyane y Sen, 2003; Sangam, 2006; Sangan y otros, 2006; López-Piñero y otros, 2008; Varaprasad y otros, 2010) y varios Premios Nobel (Kalyane y Sen, 1996; Kalyane y Kademani, 1997; Kademani y otros, 1999, 2001, 2002). En el área de Ciencia y Tecnología de Alimentos destacó Eduardo Primo Yúfera, nacido en 1918 y licenciado en Química en la Universidad de Valencia en 1941. En 1944 presentó su tesis doctoral sobre el aislamiento de nuevos tónicos cardíacos de la Digitalis thapsi L. y terminado el doctorado dedicó parte de su tiempo a la empresa industrial, desarrollando un proceso para recuperar plata metálica a partir de los líquidos de fijación de los fotógrafos y formulando productos de gran interés farmacológico.

Fue director del Departamento de Química Vegetal del Instituto de Química "Alonso Barba", perteneciente al Patronato Juan de la Cierva de Investigación Científica y Técnica del Consejo Superior de Investigaciones Científicas (CSIC). Desde este centro propició la fundación, en 1957, del Instituto de Agroquímica y Tecnología de Alimentos (IATA), del que fue director hasta 1974. Este Instituto supuso la implantación de un primer centro moderno especializado en la quími- 
ca agrícola, en la ciencia de los alimentos y en la industria alimentaria en todos y cada uno de sus principales aspectos: químico, bioquímico, microbiológico, económico y tecnológico y de competencia internacional (Aupí y Brines, 1994).

En 1961 fundó la Revista de Agroquímica y Tecnología de Alimentos, de la que fue director hasta 1977, revista que pronto fue conocida en los centros de investigación de alimentos de todo el mundo.

Desde 1974 hasta 1977 fue presidente del CSIC. Tras este período, inició una nueva etapa investigadora orientada a la ecología química. En 1990 se incorporó como investigador al Instituto de Tecnología Química (ITQ), centro mixto de la Universidad Politécnica de Valencia y el CSIC, con la novedosa estrategia de contribuir con la ciencia al desarrollo de nuevos conocimientos en ámbitos de interés industrial y su transferencia a las empresas (Castro y Fernández, 2007). Su última etapa profesional, que duró hasta 2007, año de su fallecimiento, la realizó en el Centro de Ecología Química Agrícola (CEQA), creado en 2001 como consecuencia de la importancia que habían alcanzado las investigaciones sobre Ecología Química en el ITQ.

Primo Yúfera generó a su alrededor un importante espíritu de trabajo, potenciando nuevas generaciones de investigadores e impulsando, bien él o sus discípulos, nuevos centros de investigación, no solo en la Comunidad Valenciana sino también en todo el territorio nacional. Durante su etapa como presidente del CSIC, promovió reformas orientadas a la modernización de las estructuras de investigación: cabe destacar su relevante papel en la promoción de la astrofísica, dentro de la cual, la consecución del Observatorio Astronómico de Roque de los Muchachos, en la isla de La Palma, supuso un hito para la ciencia en España (Ayala-Gascón y otros, 2011).

Le fueron concedidos una larga lista de honores y condecoraciones, entre los que cabe destacar el haber sido elegido Miembro de Honor de la Real Academia Nacional de Farmacia, y que culminaron con el más alto reconocimiento que este país da a sus tecnólogos: el Premio Nacional de Investigación Tecnológica Torres Quevedo (1988). Y, más recientemente, el Premio Rey Jaime I a las Nuevas Tecnologías (2001) (Conejero y otros, 2008). Formó parte de la junta de gobierno de la International Union of Food Science and Technology (IUFoST).

Entre sus logros científicos más reconocidos destaca: ser el primero en España en sintetizar el PAS (ácido p-amino salić́lico), primer producto eficaz contra la tuberculosis, así como desarrollar la fórmula del Alubifar (patente $\mathrm{n}^{\circ}$ 159.018) del grupo de los medicamentos antiácidos, creando sus correspondentes plantas de producción; realizar investigación básica de aplicación práctica a la industria agroalimentaria, entre las que destacan los estudios sobre las adulteraciones en zumos, calidades del arroz y aprovechamiento de sus subproductos, procesos correctores en conservas, procesos de panificación y creación del pan precocido, y en su última etapa, realizar estudios destinados al desarrollo de nuevas fórmulas para la obtención de insecticidas ecológicos, consiguiendo 7 patentes.

El sentido práctico de la investigación, el entender la investigación como solución de problemas, lo refleja en estudios cuya clara finalidad es la mejora del 
entorno, y más concretamente del entorno de la Comunidad Valenciana. De la investigación básica entendía a la perfección su importantísima función en el engranaje común de la ciencia y la economía (Ayala-Gascón, 2011). Es por ello que se le reconoce como precursor del modelo de sistema de innovación I+D+i.

\section{Objetivo}

El objetivo de este trabajo es analizar la trayectoria científica de Primo Yúfera mediante indicadores bibliométricos de productividad, colaboración e impacto. Estos indicadores permitirán identificar y caracterizar el papel que jugó en el avance de la ciencia en general y de algunas de sus áreas de conocimiento, como la Farmacognosia, Ciencia y Tecnología de los Alimentos y Ecología Química.

\section{Material y método}

Para identificar la producción científica se consultaron las siguientes bases de datos documentales: ISI-Web of Science [v.4.10] (Science Citation Index-Expanded (SCI-Expanded) y Arts \& Humanities Citation Index (AEHCI)), CAB Abstracts, CAB Abstracts Archive, Food Science and Technology Abstracts (FSTA), Analytical Abstracts, Global Health, Medline, Agricola, Agris, CSIC:ICYT-Ciencia y Tecnología, CSIC:ISOC-Ciencias Sociales y Humanidades y SCOPUS. También se revisaron las separatas y revistas del archivo personal del investigador, así como las de las bibliotecas de la Univesidad de Valencia, de la Universidad Politécnica de Valencia y del Instituto de Agroquímica y Tecnología de Alimentos.

Posteriormente se llevó a cabo un proceso de normalización y unificación de las diferentes denominaciones de los autores e instituciones. El criterio que se siguió ante dos o más variantes de un mismo nombre consistió en comprobar la coincidencia en los lugares de trabajo de cada uno de ellos. En el caso de las instituciones, además de normalizar las diferentes variantes, se presentó el problema de que algunos registros bibliográficos incluían dentro de una única adscripción institucional dos o más instituciones (fundamentalmente en el caso de institutos de investigación vinculados a universidades). En este caso, se consideraron como instituciones diferentes todas las que se podían individualizar en cada registro bibliográfico. Para la normalización de los centros se recurrió a buscarlos en revistas, directorios de universidades y centros de investigación, así como a sus respectivas páginas web.

Los indicadores bibliométricos que se han calculado se han clasificado en tres grupos: indicadores de productividad científica, indicadores de colaboración (que incluyen las redes de coautoría) e indicadores de impacto o visibilidad.

\subsection{Indicadores de productividad científica}

Los indicadores de productividad científica permiten ofrecer una dimensión cuantitativa de la actividad investigadora, ya sea de forma absoluta o relativa si 
se ponderan los valores absolutos obtenidos en relación con otras variables. Los indicadores calculados son el número de documentos publicados por Primo Yúfera y su evolución anual, el número de documentos publicados en cada centro en el que trabajó y el coeficiente de productividad, que es la relación entre la edad en la que el autor alcanzó el $50 \%$ de su productividad y el total de años de vida productiva, contando desde el año en el que publicó el primer trabajo hasta el año en el que publicó el último (Sen y Gan, 1990).

\subsection{Indicadores de colaboración y de redes}

Con el fin de analizar los patrones de colaboración se han utilizado varios indicadores: el coeficiente de colaboración, el índice de coautoría, el índice de firmas por trabajo y el índice de autores por trabajo. El coeficiente de colaboración es el número de artículos realizados en colaboración dividido por el número total de artículos (Subramanyam, 1983). El índice de coautoría corresponde al porcentaje de documentos que ha firmado el autor en colaboración con otros agentes científicos (López-Piñero y otros, 2008). El índice de firmas por trabajo es la tasa resultante de dividir el número total de firmas en los trabajos en los que ha participado un autor entre el número total de trabajos publicados. El índice de autores por trabajo es la tasa resultante de dividir el número total de autores diferentes de los trabajos en los que ha participado un autor entre el número total de trabajos publicados. También se ha determinado el número total de firmas de los trabajos, el número de autores diferentes con los que ha colaborado y el orden de la firma del autor en los trabajos realizados en colaboración.

Mediante grafos procedentes del análisis de redes sociales (ARS) se han representado las relaciones interpersonales e interinstitucionales de colaboración que ha establecido Primo Yúfera. Este tipo de análisis permite cuantificar cuántos miembros componen una red, cuál es la intensidad de las relaciones existentes entre sus miembros y quiénes son los más relevantes de la misma (GonzálezAlcaide y otros, 2008a). Se han considerado "grandes colaboradores» a aquellos autores que han publicado 9 o más artículos en colaboración con Primo Yúfera, "colaboradores medianos» a aquellos que han publicado entre 8 y 2 trabajos en colaboración y "colaboradores transitorios» a aquellos colaboradores puntuales con los que únicamente ha firmado un trabajo en colaboración con el autor o con otros colaboradores de la red. Para realizar las representaciones gráficas de la colaboración, se han utilizado dos programas de análisis y visualización de redes: Ucinet 6 for Windows (Borgatti y otros, 2002) y Pajek 1.27 (Batagelj y Mrvar, 2001).

\subsection{Indicadores de impacto o visibilidad de los trabajos}

Los indicadores de impacto o visibilidad de los trabajos miden la repercusión que éstos han tenido en estudios posteriores y se calculan a partir del análisis 
de citas que han recibido en las revistas incluidas en Science Citation Index. Uno de los indicadores más difundidos es el factor de impacto (FI) de las revistas, publicado anualmente en el Journal Citation Reports, que mide la frecuencia media de citación de cada revista en los dos años anteriores. En la actualidad se ha convertido en un índice de calidad de las publicaciones, ya que se basa en el reconocimiento de su valor por la comunidad científica a través de las citas (Aleixandre y otros, 2007). En este trabajo se ha identificado el FI de las revistas (total y medio), el número de citas recibidas por el autor, el número de trabajos citados, el índice citas/trabajo, los artículos más citados (bot papers) y el factor $\mathrm{h}$ (h-index), que indica el número de trabajos que tiene un determinado autor con ese mismo número de citas (Hirsch, 2005). En este apartado se ha identificado también la distribución por países de edición de las revistas y según su clasificación temática.

\section{Resultados y discusión}

La tabla I presenta los principales indicadores de la productividad, colaboración e impacto de los artículos publicados. A lo largo de su carrera investigadora, que inició en 1943 y concluyó en 2007, Primo Yúfera publicó 291 trabajos de diversa tipología documental (artículos, notas y revisiones), de los que el 89\% $(n=259)$ corresponden a artículos originales. De los 291 trabajos, el 89,3\% $(n=260)$ han sido firmados en colaboración con otros autores. Ha colaborado con 161 autores diferentes y el número de coautorías totales identificadas, incluyendo las coautorías entre sus colaboradores, asciende a 1.456, siendo 696 las coautorías concernientes a este autor. En el $41,1 \%$ del total de los trabajos $(n=107)$ figura como primer firmante de los artículos. El índice firmas por trabajo (IFT) global de su etapa investigadora es de 3,4 autores por trabajo y el índice de autores distintos por trabajo se sitúa en 0,56. El número de citas recibidas, número de trabajos citados, índice citas/trabajo y el factor h (h-index) se calculó únicamente con los 86 registros recuperados en SCI el 19 de marzo de 2011 y representa únicamente un $29,55 \%$ de la totalidad de su producción científica.

El idioma de publicación prioritario de Primo Yúfera fue el español, que representa el $78 \%$ del total de los trabajos $(n=227)$, el resto de su producción fue en inglés $(n=64)$.

Los estudios realizados en el área de Ciencia y Tecnología de Alimentos fueron principalmente de ámbito regional agrícola relacionados con alimentos como el arroz, los cítricos, la chufa y la uva, entre otros, resultados que difundió a través de la Revista de Agroquímica y Tecnología de Alimentos, que se editaba en español. Aunque la revista estuvo indexada en el JCR desde 1989 hasta 1991, año en que pasó a ser la Revista Española de Ciencia y Tecnología de Alimentos, en ese período únicamente publicó 1 artículo en 1989 y 2 en el año 1990, por lo que tuvo escasa visibilidad internacional a través de citas, pero una amplia proyección internacional a través de la relaciones y convenios de colaboración que 
TABLA I

Principales indicadores de productividad, colaboración e impacto científico de Eduardo Primo Yúfera

\begin{tabular}{l|c}
\hline \multicolumn{1}{c|}{ Medidas/indicadores } & \\
\hline $\mathrm{N}^{\circ}$ de trabajos & 291 \\
\hline Coeficiente de productividad & 0,42 \\
\hline $\mathrm{N}^{\circ}$ de firmas & 987 \\
\hline $\mathrm{N}^{\circ}$ de autores distintos con los que ha colaborado & 161 \\
\hline $\mathrm{N}^{\circ}$ de trabajos firmados por dos o más autores (\%) & $260(89,30 \%)$ \\
\hline Coeficiente de colaboración & 0,89 \\
\hline $\mathrm{N}^{\circ}$ de coautorías de Eduardo Primo Yúfera & 696 \\
\hline $\mathrm{N}^{\circ}$ de coautorías incluyendo las coautorías entre sus colaboradores & 1.456 \\
\hline $\mathrm{N}^{\circ}$ de artículos como único autor (\%) & $31(10,70 \%)$ \\
\hline $\mathrm{N}^{\circ}$ de artículos como primer firmante (\%) & $107(41,10 \%)$ \\
\hline Índice firmas/trabajo (IFT) & 3,4 \\
\hline Índice autores/trabajo & 0,56 \\
\hline $\mathrm{N}^{\circ}$ de citas recibidas* & 482 \\
\hline $\mathrm{N}^{o}$ de trabajos citados (\%)* & $67(77,9 \%)$ \\
\hline Índice citas/trabajo* & 7,19 \\
\hline Factor h (h-index)* & 12 \\
\hline
\end{tabular}

* Estos índices corresponden únicamente a los 86 registros indexados en el Science Citation Index (SCI).

estableció el IATA con países como Cuba, México, Argentina, Chile, Bolivia, Perú, Costa Rica, China y EEUU.

\subsection{Productividad científica}

La tabla II presenta la distribución temporal de la productividad científica por quinquenios diferenciando los trabajos firmados como único autor y los firmados en colaboración. Su primer artículo se publicó en 1943 y el último en 2007.

A lo largo de su trayectoria investigadora publicó una media de 4,48 trabajos anuales. Desde 1943 hasta 1962 se observa una productividad científica ascendente, que casi duplica cada etapa anterior. La década de los sesenta supuso el período de máxima productividad: así, en el quinquenio 1963-1967 publicó 56 trabajos y 51 en el quinquenio 1968-1972, lo que supone una media de 10,7 trabajos anuales. En 1969 alcanzó el 50\% de su producción científica. A partir del año 1975, su productividad científica comenzó a disminuir, coincidiendo con un cambio en su trayectoria profesional, abandonando temporalmente la investigación para ocupar la presidencia del Consejo Superior de Investigaciones Cientí- 
TABLA II

Productividad del autor Eduardo Primo Yúfera

\begin{tabular}{c|c|c|c|c}
\hline Quinquenio & $\begin{array}{c}\mathbf{N}^{\mathbf{o}} \text { de artículos } \\
\text { como único } \\
\text { autor }\end{array}$ & $\begin{array}{c}\mathbf{N}^{\mathbf{o}} \text { de artículos } \\
\text { en colaboración }\end{array}$ & $\begin{array}{c}\mathbf{N}^{\circ} \text { total } \\
\text { de artículos }\end{array}$ & $\begin{array}{c}\mathbf{N}^{\mathbf{a}} \text { acumulado } \\
\text { de artículos }\end{array}$ \\
\hline $1943-1947$ & 2 & 4 & 6 & 6 \\
\hline $1948-1952$ & 1 & 10 & 11 & 17 \\
\hline $1953-1957$ & 0 & 21 & 21 & 38 \\
\hline $1958-1962$ & 4 & 34 & 38 & 76 \\
\hline $1963-1967$ & 3 & 53 & 56 & 132 \\
\hline $1968-1972$ & 12 & 39 & 51 & 183 \\
\hline $1973-1977$ & 3 & 18 & 21 & 204 \\
\hline $1978-1982$ & 1 & 7 & 8 & 229 \\
\hline $1983-1987$ & 2 & 15 & 17 & 240 \\
\hline $1988-1992$ & 3 & 8 & 11 & 260 \\
\hline $1993-1997$ & 0 & 20 & 20 & 287 \\
\hline $1998-2002$ & 0 & 27 & 27 & 291 \\
\hline $2003-2007$ & 0 & 4 & 4 & \\
\hline
\end{tabular}

ficas, cargo que ejerció desde 1974 hasta 1977. Tras su cese en esta institución y su reincorporación a las tareas de investigación, se produjo un cambio en sus líneas de investigación, pasando del ámbito de la ciencia y tecnología de alimentos al de la ecología química, de manera que a partir del 1983 comenzó una ascensión que se prolongó hasta el año 2002.

La tabla III recoge el número de trabajos firmados en cada una de las instituciones en las que realizó su actividad investigadora. Publicó 103 trabajos desde el Instituto de Agroquímica y Tecnología de los Alimentos (IATA) y 93 en el Departamento de Química Vegetal del Instituto de Química Alonso Barba; teniendo en cuenta que éste último derivó en el IATA, prácticamente el $40 \%$ de sus trabajos corresponden a este Instituto. Estando trabajando en el Departamento de Química Orgánica, Bioquímica y Química Agrícola, también aportó un número elevado de trabajos, que representó el 53,8\% de los trabajos realizados en la Universidad Politécnica de Valencia. Un porcentaje elevado de sus trabajos corresponden al Instituto de Tecnología Química, centro mixto del CSIC-UPV, y al Departamento de Química Orgánica de la Universidad de Valencia, reuniendo cada uno de ellos el 9,7\% y el 9,9\% de los trabajos, respectivamente.

La figura 1 muestra, a lo largo de trece quinquenios, la distribución de las publicaciones de Primo Yúfera, así como el número acumulado de trabajos, diferenciando las publicaciones realizadas como único autor o en colaboración y los coeficientes de colaboración correspondientes. La mayor productividad se 
TABLA III

Número de trabajos publicados por Eduardo Primo Yúfera en cada centro de trabajo

\begin{tabular}{l|l|r}
\hline Institución & \multicolumn{1}{|c}{ Centro/Departamento } & Total \\
\hline UV & Laboratorio de Química Orgánica & 48 \\
\hline \multirow{4}{*}{ CSIC } & Instituto José Celestino Mutis de Farmacognosia & 11 \\
\cline { 2 - 3 } & Instituto de Química Alonso Barba-Departamento de Química Vegetal & 93 \\
\cline { 2 - 3 } & Instituto de Agroquímica y Tecnología de los Alimentos (IATA) & 103 \\
\hline CSIC-UPV & Instituto de Tecnología Química (ITQ) & 47 \\
\hline \multirow{5}{*}{ UPV } & Departamento de Química Orgánica, Bioquímica y Química Agrícola & 100 \\
\cline { 2 - 3 } & Departamento de Química, Bioquímica y Microbiología & 3 \\
\cline { 2 - 3 } & Departamento de Bioquímica y Química Agrícola & 17 \\
\cline { 2 - 3 } & Departamento de Biotecnología & 63 \\
\cline { 2 - 3 } & Centro de Ecología Química Agrícola (CEQA) & 3 \\
\hline
\end{tabular}

FIGURA 1

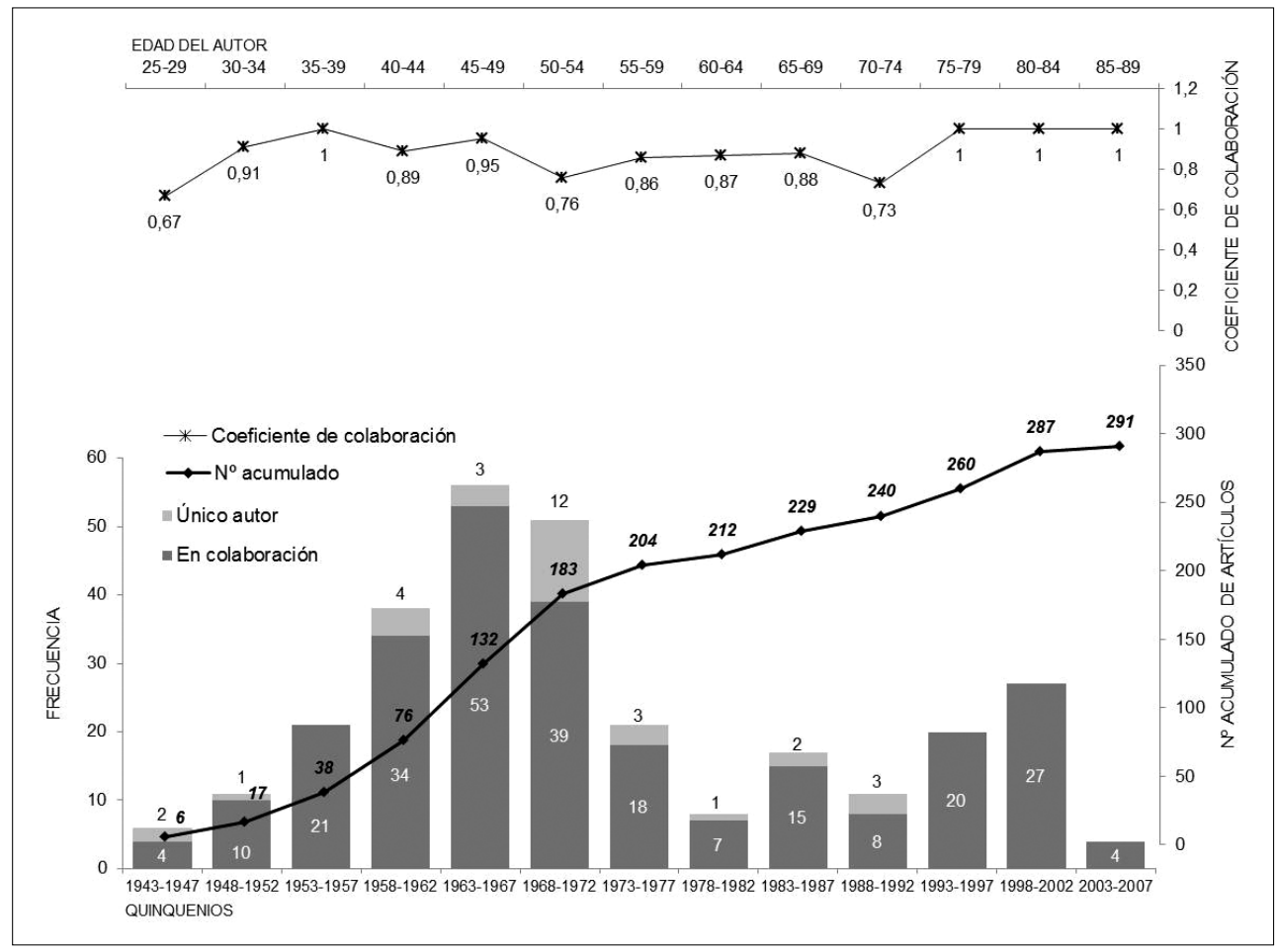


produjo en 1962 con 17 trabajos (a la edad de 44 años), seguido de 14 trabajos en 1963 (a la edad de 45 años) y 13 trabajos en 1969 (a la edad de 51 años). Sin embargo, el período más productivo de su vida fue de los 40 a los 54 años. El 50\% de su productividad la alcanzó a la edad de 51 años, tras 27 de actividad. La vida productiva del autor fue de 65 años y empezó cuando el investigador tenía 25, por lo que el coeficiente de productividad es de 0,42 (resultado de dividir 27 años entre 65 años).

\subsection{Patrones de colaboración}

El 89,3\% de los artículos de Primo Yúfera fueron realizados en colaboración, firmando el 100\% de sus artículos en colaboración durante los períodos 19531957 y 1993-2007 (figura 1).

La tabla IV recoge el orden o posición de la firma de los trabajos. En 138 trabajos $(47,4 \%)$ fue primer firmante, pero si se tiene en cuenta únicamente el conjunto de trabajos realizados en colaboración, aparece como primer firmante en el $41,2 \%$ de los trabajos ( $n=107$ ), como último firmante en el $45 \%$ de los trabajos $(n=117)$ y en el $13,8 \%$ de los trabajos $(n=36)$ su firma aparece en posiciones intermedias.

\section{TABLA IV}

Autoría de Eduardo Primo Yúfera

\begin{tabular}{|c|c|c|c|c|c|c|c|c|}
\hline \multirow[b]{2}{*}{$N^{o}$ de autores } & \multicolumn{7}{|c|}{$\mathrm{N}^{\circ}$ de artículos agrupados por la posición del autor } & \multirow[b]{2}{*}{ Total } \\
\hline & $\begin{array}{c}\text { 1er } \\
\text { autor }\end{array}$ & $\begin{array}{c}2^{\circ} \\
\text { autor }\end{array}$ & $\begin{array}{c}\text { 3er } \\
\text { autor }\end{array}$ & $\begin{array}{c}4^{\circ} \\
\text { autor }\end{array}$ & $\begin{array}{c}5^{\circ} \\
\text { autor }\end{array}$ & $\begin{array}{c}\mathbf{6}^{\mathbf{0}} \\
\text { autor }\end{array}$ & $\begin{array}{c}7^{\circ} \\
\text { autor }\end{array}$ & \\
\hline Único autor & 31 & & & & & & & 31 \\
\hline 2 autores & 18 & 14 & & & & & & 32 \\
\hline 3 autores & 49 & 6 & 43 & & & & & 98 \\
\hline 4 autores & 31 & 4 & 6 & 38 & & & & 79 \\
\hline 5 autores & 6 & 0 & 2 & 9 & 15 & & & 32 \\
\hline 6 autores & 2 & 0 & 0 & 1 & 6 & 4 & & 13 \\
\hline 7 autores & 1 & 1 & 0 & 0 & 0 & 0 & 3 & 5 \\
\hline 9 autores & 0 & 0 & 1 & 0 & 0 & 0 & 0 & 1 \\
\hline Total & 138 & 25 & 52 & 48 & 21 & 4 & 3 & 291 \\
\hline
\end{tabular}

La figura 2 muestra como evolucionó su firma con los años, pasando de primera a última posición, en el período comprendido entre 1943 y 1972 la firma aparece predominantemente en la primera posición y entre 1973 y 2007 aparece predominantemente en la última posición, coincidiendo este cambio en la ten- 
dencia con el cambio en la temática de sus investigaciones. En el primer período la temática predominante de sus publicaciones es la Ciencia y Tecnología de los Alimentos y en el segundo período es la Ecología Química.

FIGURA 2

Evolución de la firma en los artículos de Eduardo Primo Yúfera

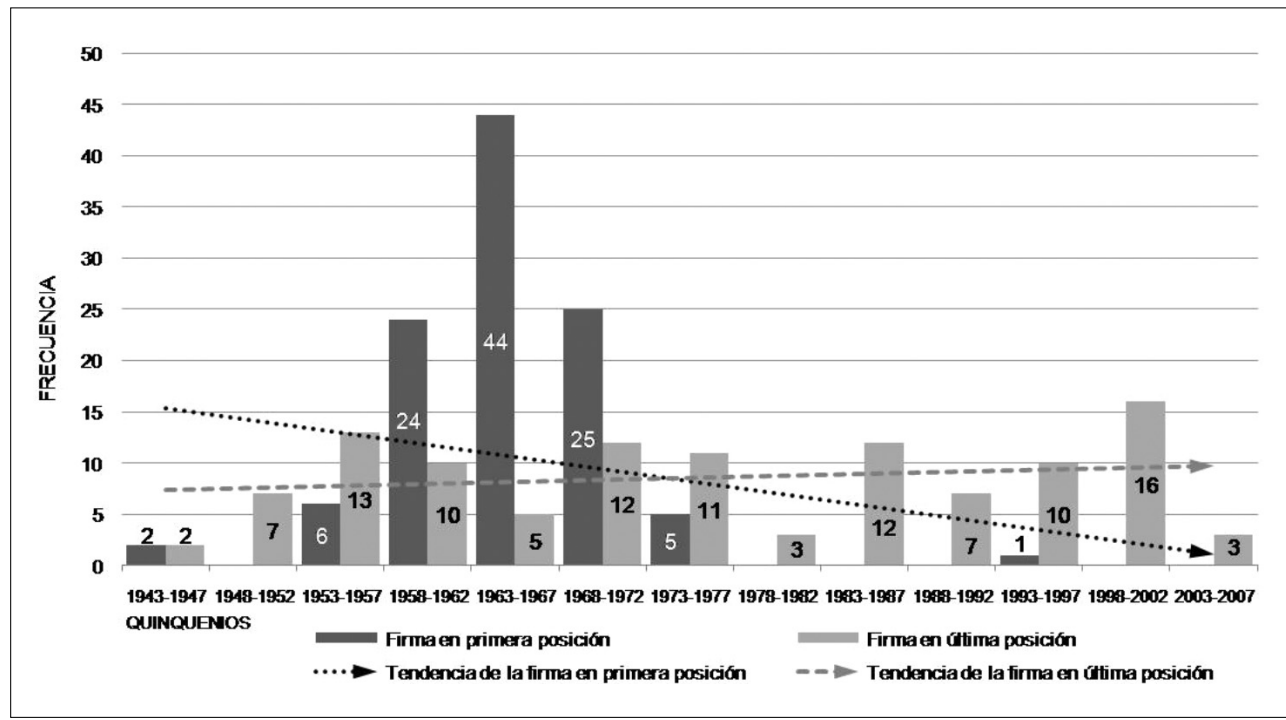

La tabla $\mathrm{V}$ presenta la distribución quinquenal de los investigadores que colaboraron en sus trabajos, el número de trabajos realizadas en colaboración con cada uno de ellos y el número total de colaboradores en cada período. En el quinquenio 1963-1967 se produjeron el mayor número de colaboraciones $(n=169)$, realizadas por 36 autores diferentes, seguido del quinquenio 1968-1972 ( $n=153$ ), en el que fueron realizadas por 37 autores diferentes. Veinticinco años después aparece otro período de elevadas colaboraciones (1998-2002), reuniendo 137, y entre 1958-1962 se alcanzaron 119 colaboraciones.

Si se analizan las colaboraciones separadamente en cada uno de los quinquenios, los principales colaboradores fueron Casas Carramiñana, con quien publicó 15 trabajos entre los años 1958-1962, y Lafuente Ferriols (1963-1967), Bello Sáez y Esplugues Requena (1993-1997), con quienes publicó 13 artículos con cada uno de ellos.

El $51,9 \%$ de las colaboraciones $(n=361)$ las aportaron 20 autores, firmando cada uno más de 9 trabajos con Primo Yúfera; 50 autores colaboraron entre 8 y 3 veces, reuniendo el 31,2\% de las colaboraciones $(n=217)$, el 16,9\% restante $(n=118)$ corresponden a 27 autores que realizaron 2 colaboraciones y 64 autores que colaboraron puntualmente, con los que únicamente firmó un traba- 


\section{TABLA V}

\section{Distribución quinquenal de los colaboradores de Eduardo Primo Yúfera}

\begin{tabular}{|c|c|c|}
\hline Período & Colaboradores con número de artículos & Total \\
\hline $1943-1947$ & Costa Novella, E(3); Ribas, I(1); Sánchez, A(1) & 3 \\
\hline $1948-1952$ & $\begin{array}{l}\text { Beltrán Bigorra, F(1); Caridad, A(1); Casabuena Puig, A(1); Colom Pizá, J(1); } \\
\text { Costa Novella, E(1); Lafuente Ferriols, B(2); Miralles Jiménez de la Espada, } \\
\text { L(1); Navarro Botella, R(1); Santa Pau Vota, A(3);Viguera Lobo, JM(6) }\end{array}$ & 10 \\
\hline $1953-1957$ & $\begin{array}{l}\text { Alcalá Colombrí, A(3); Casas Carramiñana, A(9); Castillo, P(1); Colom Pizá, } \\
\text { J(2); Cortés Navarro, V(2); García Álvarez, R(1); Gómez Fabra, JL(2); La- } \\
\text { fuente Ferriols, B(6); López Fernández, J(2); Navarro Botella, R(1); Nebot } \\
\text { Gil, E(1); Pascual Raga, F(3); Pla Jiménez, J(3); Royo Iranzo, J(6); Tamm, } \\
\text { C(1); Tarazona Martínez, V(2); Verdeguer Sirera, J(1); Viguera Lobo, JM(5) }\end{array}$ & 18 \\
\hline $1958-1962$ & $\begin{array}{l}\text { Alberola Matoses, J(1); Barber Pérez, S(7); Benedito de Barber, C(5); Botella } \\
\text { Soto, C(2); Calvo Gutiérrez-Ravé, C(1); Cánovas, F(1); Carrasco Andreu, } \\
\text { A(3); Casas Carramiñana, A(15); Cortés Navarro, V(6); Cuñat Broseta, P(3); } \\
\text { De Oyanguren, J(1); García Álvarez, R(1); Gasqué Pastor, F(3); Hernández } \\
\text { Giménez, E(2); Koen Mosse, J(5); Lafuente Ferriols, B(11); Lorenz Gil, A(1); } \\
\text { Piñaga Otamendi, F(1); Planelles, V(1); Royo Iranzo, J(10); Sala Gomis, JM(1) }\end{array}$ & 21 \\
\hline $1963-1967$ & $\begin{array}{l}\text { Alberola Matoses, J(4); Asensi, P(1); Barber Pérez, S(12); Benedito de Barber, } \\
\text { C(8); Benedito López, J(1); Calvo Gutiérrez-Ravé, C(2); Cañizares Sales, } \\
\text { J(1); Carrasco Andreu, A(1); Carrasco Dorrien, JM(1); Casas Carramiñana, } \\
\text { A(8); Cohenca, JM(1); Cuñat Broseta, P(11); Durán Hidalgo, L(4); Flores } \\
\text { Durán, J(1); García Álvarez, R(1); Gasqué Pastor, F(5); Hernández Giménez, } \\
\text { E(3); Hernández Yago, J(1); Hernández, D(2); Koen Mosse, J(1); Lafuente } \\
\text { Ferriols, B(13); Mallent, D(2); Martínez Silvestre, RM(1); Miralles, MC(1); } \\
\text { Montesinos Jarque, M(1); Moreno Royo, L(1); Munárriz, JM(1); Piñaga } \\
\text { Otamendi, F(3); Rodrigo Enguídanos, M(1); Royo Iranzo, J(5); Sala Gomis, } \\
\text { JM(6); Sánchez Paradeda, J(3); Serra Sister, J(1); Tortosa Martorell, E(2); } \\
\text { Vayá, JL(2); Villagrasa, J(1) }\end{array}$ & 36 \\
\hline $1968-1972$ & $\begin{array}{l}\text { Alberola Matoses, J(4); Barber Pérez, S(5); Benedito de Barber, C(4); Bermell, } \\
\text { AM(2); Camacho, J(1); Carrasco Dorrien, JM(7); Casaña Giner, V(2); Casas } \\
\text { Carramiñana, A(3); Catalá Moragrera, R(1); Catalá, E(1); Chamarro Lapuer- } \\
\text { ta, J(3); Cornejo, MP(1); Cuñat Broseta, P(10); Durán Hidalgo, L(3); Flores } \\
\text { Durán, J(2); Hernández Giménez, E(1); Hernández Yago, J(3); Jiménez, } \\
\text { A(1); Lafuente Ferriols, B(8); Mallent, D(1); Martínez Silvestre, RM(2); Mar- } \\
\text { tínez, M(4); McLean, N(1); Montoro Martínez, R(1); Pérez Pastor, R(2); } \\
\text { Piñaga Otamendi, F(6); Rivero, M(1); Romero Villafranca, R(4); Royo Iran- } \\
\text { zo, J(3); Sala Gomis, JM(6); Sánchez, L(2); Tomás, J(2); Tortosa Martorell, } \\
\text { E(1); Ull de Molins, J(1); Vayá, JL(1); Vega, R(1); Vila, R(1) }\end{array}$ & 37 \\
\hline 1973-1977 & $\begin{array}{l}\text { Abadía, AM(1); Barber Pérez, S(1); Beltrán Porter, JP(1); Benedito López, J(2); } \\
\text { Bermell, AM(1); Blanco, MC(1); Calvo Gutiérrez-Ravé, C(1); Carbonell Ta- } \\
\text { lón, JV(1); Carrasco Dorrien, JM(2); Casas Carramiñana, A(2); Conejero } \\
\text { Tomás, V(2); Cuñat Broseta, P(5); Durán Hidalgo, L(1); Flores Durán, J(2); } \\
\text { Flores Pedauye, R(1); Garro, R(2); Gasqué Pastor, F(1); González de Bui- } \\
\text { trago Pla, M(1); Hernández Giménez, E(2); Jorro, M(1); Lafuente Ferriols, } \\
\text { B(3); Martínez, M(2); Miro, E(2); Montoro Martínez, R(1); Piñaga Otamen- } \\
\text { di, F(4); Ribó Canut, J(1); Sendra Sena, J(1); Tadeo Lluch, JL(1); Vila, R(1) }\end{array}$ & 29 \\
\hline
\end{tabular}




\section{TABLA V (continuación)}

\begin{tabular}{|c|c|c|}
\hline Período & Colaboradores con número de artículos & Total \\
\hline 1978-1982 & $\begin{array}{l}\text { Castelló, F(1); Culiañez Maciá, FA(1); Cuñat Broseta, P(1); Gil Tortosa, CI(1); } \\
\text { Grino, J(1); Guerri Sirera, J(1); Legaz Paredes, F(1); Maquieira Catalá, A(1); } \\
\text { Martínez Pardo, R(1); Pazos, JM(1); Primo Millo, E(2); Ribas, I(2); Ribó } \\
\text { Canut, J(3); Romero Villafranca, R(1); Rubio Delgado, JL(1); Sala Gomis, } \\
\text { JM(1); Sueiras Romero, J(2) }\end{array}$ & 17 \\
\hline 1983-1987 & $\begin{array}{l}\text { Aliaga Morell, JR(1); Benedito de Barber, C(3); Clemente Marín, G(3); Climent } \\
\text { Morató, MD(1); Cordero Contreras, V(3); Culiañez Maciá, FA(2); Cuñat } \\
\text { Broseta, P(1); De Miguel García, P(1); García Breijo, FJ(2); Ginesta Peris, } \\
\text { E(1); Guerri Sirera, J(1); Hernández Yago, J(1); Llorens, J(1); Maquieira } \\
\text { Catalá, A(3); Primo Millo, E(1); Puchades Pla, R(5); Romero Villafranca, } \\
\text { R(1); Rubio Delgado, JL(3); Sanz Berzosa, I(1) }\end{array}$ & 19 \\
\hline 1988-1992 & $\begin{array}{l}\text { Bowers, WS(1); Casas Carramiñana, A(1); Chiralt Boix, A(1); Cuñat Broseta, } \\
\text { P(1); De Miguel García, P(1); Garcerá Zamorano, MD(1); García Breijo, } \\
\text { FJ(2); Gil Tortosa, CI(1); Guardiola Herráez, VG(1); March Iborra, MC(2); } \\
\text { Martínez Pardo, R(1); Sanz Berzosa, I(4) }\end{array}$ & 12 \\
\hline 1993-1997 & $\begin{array}{l}\text { Adelantado Mateu, M(1); Ballach Parisi, S(1); Barrachina Sancho, MD(7); Be- } \\
\text { llo Saez, R(13); Beltrán Niclós, B(2); Borges, C(1); Calatayud, S(1); Castillo } \\
\text { López, MA(1); Couillaud, F(1); Cuñat Broseta, P(1); Esplugues Mota, JV(1); } \\
\text { Esplugues Requena, J(13); Garcerá Zamorano, MD(1); García Breijo, FJ(2); } \\
\text { Gil Tortosa, CI(1); Ginesta Peris, E(2); Guerri Sirera, J(1); Hernández Ala- } \\
\text { mos, MM(1); Legaz Paredes, F(1); López de Medrano Villar, MJ(3); Ma- } \\
\text { quieira Catalá, A(1); Martínez Cuesta, MA(5); Martínez Mañez, R(1); Mar- } \\
\text { tínez Pardo, R(1); Miranda Alonso, MA(1); Moreno Royo, L(5); Moya Sanz, } \\
\text { P(1); Muñoz, N(1); Primo Millo, J(3); Puchades Pla, R(1); Raigón Jiménez, } \\
\text { MD(1); Ruiz, L(1); Sanz Berzosa, I(2) }\end{array}$ & 33 \\
\hline 1998-2002 & $\begin{array}{l}\text { Adelantado Mateu, M(1); Álvarez Manilla, G(1); Bello Saez, R(9); Beltrán } \\
\text { Niclós, B(7); Calatayud, S(7); Cantín Sanz, A(7); Casaña Giner, V(4); Cas- } \\
\text { tillo López, MA(4); Corma Canós, A(1); Esplugues Requena, J(9); Gandía } \\
\text { Balaguer, A(3); Garrido Vivas, A(1); Hernández Alamos, MM(2); Hernán- } \\
\text { dez Giménez, E(2); Hernández Sánchez, G(1); Lull Noguera, C(1); Ma- } \\
\text { quieira Catalá, A(1); Martínez Téllez, MA(2); Mengod Puerta, C(2); Miran- } \\
\text { da Alonso, MA(7); Moreno Royo, L(6); Moya Sanz, P(9); Muñoz Pallares, } \\
\text { J(1); Primo Millo, J(10); Puchades Pla, R(1); Raigón Jiménez, MD(1); Ri- } \\
\text { vera Castaneda, G(1); Sanz Berzosa, I(4); Vallejo Cohen, S(1); Vargas } \\
\text { Arispuro, I(4) }\end{array}$ & 30 \\
\hline 2003-2007 & $\begin{array}{l}\text { Almada Ruiz, E(1); Ayala Mingol, I(2); Casaña Giner, V(1); González Martínez, } \\
\text { MC(1); Hernández Alamos, MM(1); Lull Noguera, C(1); Martínez Téllez, } \\
\text { MA(1); Moya Sanz, P(1); Navarro Llopis, V(2); Primo Millo, J(2); Sanchis } \\
\text { Cabanes, J(2); Vallejo Cohen, S(1); Vargas Arispuro, I(1) }\end{array}$ & 13 \\
\hline
\end{tabular}

jo en colaboración. Lafuente Ferriols es el autor que más trabajos publicó en colaboración con Primo Yúfera, con 43 artículos, seguido de Casas Carramiñana con 37, Cuñat Broseta con 33, Barber Pérez con 25 y Royo Iranzo con 24 (tabla VI). 
TABLA VI

Principales colaboradores de Eduardo Primo Yúfera (más de 9 trabajos firmados en coautoría), institución, periodo de colaboración $y n^{o}$ de colaboraciones

\begin{tabular}{|c|c|c|c|}
\hline Nombre del autor & Institución & Período & $\begin{array}{l}\mathbf{N}^{\circ} \text { de } \\
\text { artículos }\end{array}$ \\
\hline Lafuente Ferriols, B. & CSIC-IATA & $1952-1975$ & 43 \\
\hline Casas Carramiñana, A. & CSIC-IATA & 1953-1990 & 37 \\
\hline Cuñat Broseta, $\mathrm{P}$. & CSIC-IATA & 1961-1994 & 33 \\
\hline Barber Pérez, S. & CSIC-IATA & $1958-1976$ & 25 \\
\hline Royo Iranzo, J. & CSIC-IATA & $1956-1971$ & 24 \\
\hline Bello Sáez, R. & $\begin{array}{l}\text { UV-Dept. de Farmacología } \\
\text { Fundación Juan Esplugues }\end{array}$ & 1995-2002 & 22 \\
\hline Esplugues Requena, J. & $\begin{array}{l}\text { UV-Dept. de Farmacología } \\
\text { Fundación Juan Esplugues }\end{array}$ & 1995-2002 & 22 \\
\hline Benedito de Barber, C. & CSIC-IATA & 1962-1986 & 20 \\
\hline Primo Millo, J. & CSIC-UPV-ITQ /UPV-CEQA & 1994-2003 & 15 \\
\hline Piñaga Otamendi, F. & CSIC-IATA & $1962-1975$ & 14 \\
\hline Sala Gomis, J. M. & CSIC-IATA & 1962-1980 & 14 \\
\hline Moreno Royo, L. & $\begin{array}{l}\text { UV-Dept. de Farmacología } \\
\text { Fundación Juan Esplugues }\end{array}$ & 1963-2002 & 12 \\
\hline Moya Sanz, P. & CSIC-UPV-ITQ /UPV-CEQA & $1997-2003$ & 11 \\
\hline Sanz Berzosa, I. & CSIC-UPV-ITQ & 1986-2001 & 11 \\
\hline Viguera Lobo, J. M. & $\begin{array}{l}\text { UV-Dept. de Química Orgánica } \\
\text { CSIC-IATA }\end{array}$ & $1950-1954$ & 11 \\
\hline Carrasco Dorrien, J. M. & CSIC-IATA & $1967-1976$ & 10 \\
\hline Hernández Giménez, E. & CSIC-UPV-ITQ /UPV-CEQA & $1961-2000$ & 10 \\
\hline Alberola Matoses, J & CSIC-IATA & $1962-1971$ & 9 \\
\hline Beltrán Niclós, B. & $\begin{array}{l}\text { UV-Dept. de Farmacología } \\
\text { Fundación Juan Esplugues }\end{array}$ & 1997-2001 & 9 \\
\hline Gasqué Pastor, F. & CSIC-IATA & 1962-1974 & 9 \\
\hline
\end{tabular}

Cabe destacar que la mayor colaboración se produjo con miembros del Instituto de Agroquímica y Tecnología de las Alimentos (IATA) (70,6\%). El 18,0\% de las colaboraciones fueron con miembros del Departamento de Farmacología de la Universidad de Valencia, seguido de un 10,5\% de colaboraciones con los miembros del Instituto de Tecnología Química y, en último lugar, con los miem- 
bros del Centro de Ecología Química Agrícola (CEQA) (0,9\% de colaboraciones). Cabe destacar que con Hernández Giménez y Moreno Royo estuvo colaborando durante un período de 40 años y 38 años con Casas Carramiñana.

Los análisis de redes sociales basados en las coautorías de trabajos científicos sólo han sido objeto de estudio recientemente (Newman, 2001), entre los que caben destacar varios artículos de las áreas de ciencias de la salud y de sociología (González-Alcaide y otros, 2006, 2008a, 2008b, 2009; Valderrama-Zurián y otros, 2007; Aleixandre-Benavent y otros, 2008). En las figuras 3 y 4 se muestran las redes de colaboradores de Primo Yúfera desglosadas en dos períodos que se corresponden con la temática dominante de sus publicaciones: desde 1943 hasta 1977, en el que predominan las publicaciones del área del Ciencia y Tecnología de los Alimentos y desde 1978 hasta 2007, en el que predominan las publicaciones relacionadas con el área de Ecología Química. Los colaboradores transitorios, es decir, aquellos con los que publicó un único trabajo en colaboración, no aparecen representados en la red, a fin de facilitar una visualización más clara de las redes de colaboración y centrar el análisis en las relaciones de colaboración más intensas. La intensidad de la colaboración viene definida por un trazo más grueso en los enlaces entre dos colaboradores. Además, sobre cada línea se indica el número de colaboraciones entre los dos autores unidos por la línea.

La figura 3 refleja la red de colaboradores de Eduardo Primo Yúfera en el período 1943-1977. En esta etapa colaboró con 95 autores diferentes (11 grandes colaboradores, 37 colaboradores medianos y 47 colaboradores transitorios). Predominaron las colaboraciones con un máximo de tres a cuatro autores, siendo Lafuente Ferriols, Casas Carramiñana, Royo Iranzo, Barber Pérez, Benedito de Barber y Cuñat Broseta los autores con los que ha mantenido un mayor número de colaboraciones. Cabe destacar la intensa colaboración que también mantienen entre sí los colaboradores de Primo Yúfera. Aparecen definidos 6 subgrupos de colaboradores pero muy interrelacionados entre ellos, que se traduce en un importante intercambio del conocimiento entre grupos dedicados a diferentes ámbitos investigación dentro del área de la Ciencia y Tecnología de los Alimentos.

La figura 4 muestra la red de colaboradores a lo largo del período 19782007, en el que la temática predominante de las publicaciones es la Ecología Química. En este período también mantuvo relaciones de colaboración muy intensas aunque con un menor número de autores. Publicó trabajos científicos con 77 colaboradores ( 7 grandes colaboradores, 43 colaboradores medianos y 27 colaboradores transitorios). En este período se identifican tres grandes subgrupos de colaboradores, uno de ellos está formado por 8 miembros, liderado por Bello Saez y Esplugues Requena. Otro subgrupo está integrado por 10 miembros, con Primo Millo y Moya Sanz, como miembros más destacados e interconectado a través de este último con Vargas Arispuro, que forma parte del otro subgrupo formado por 7 miembros, y que tiene como autor destacado a Sanz Berzosa. 


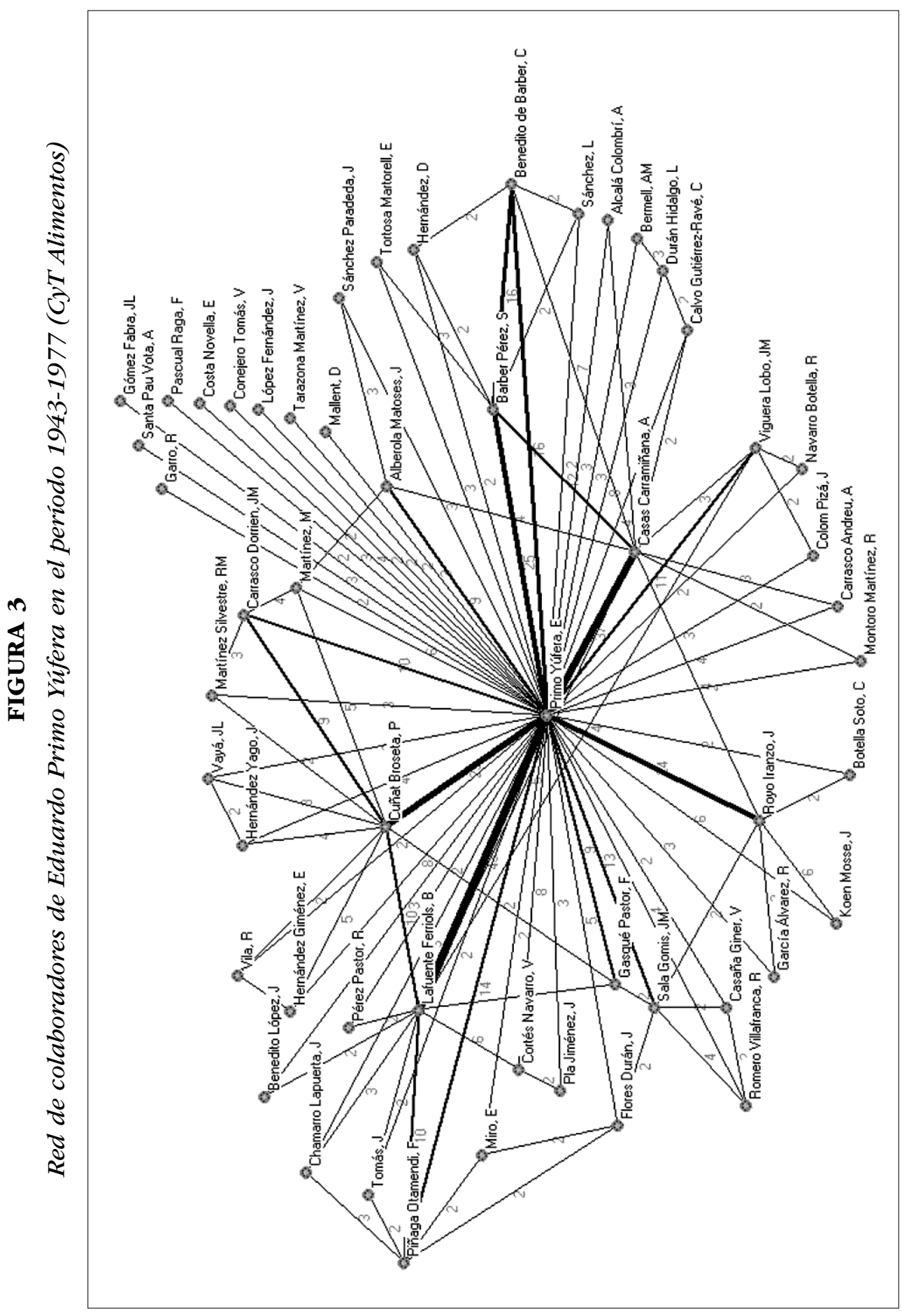




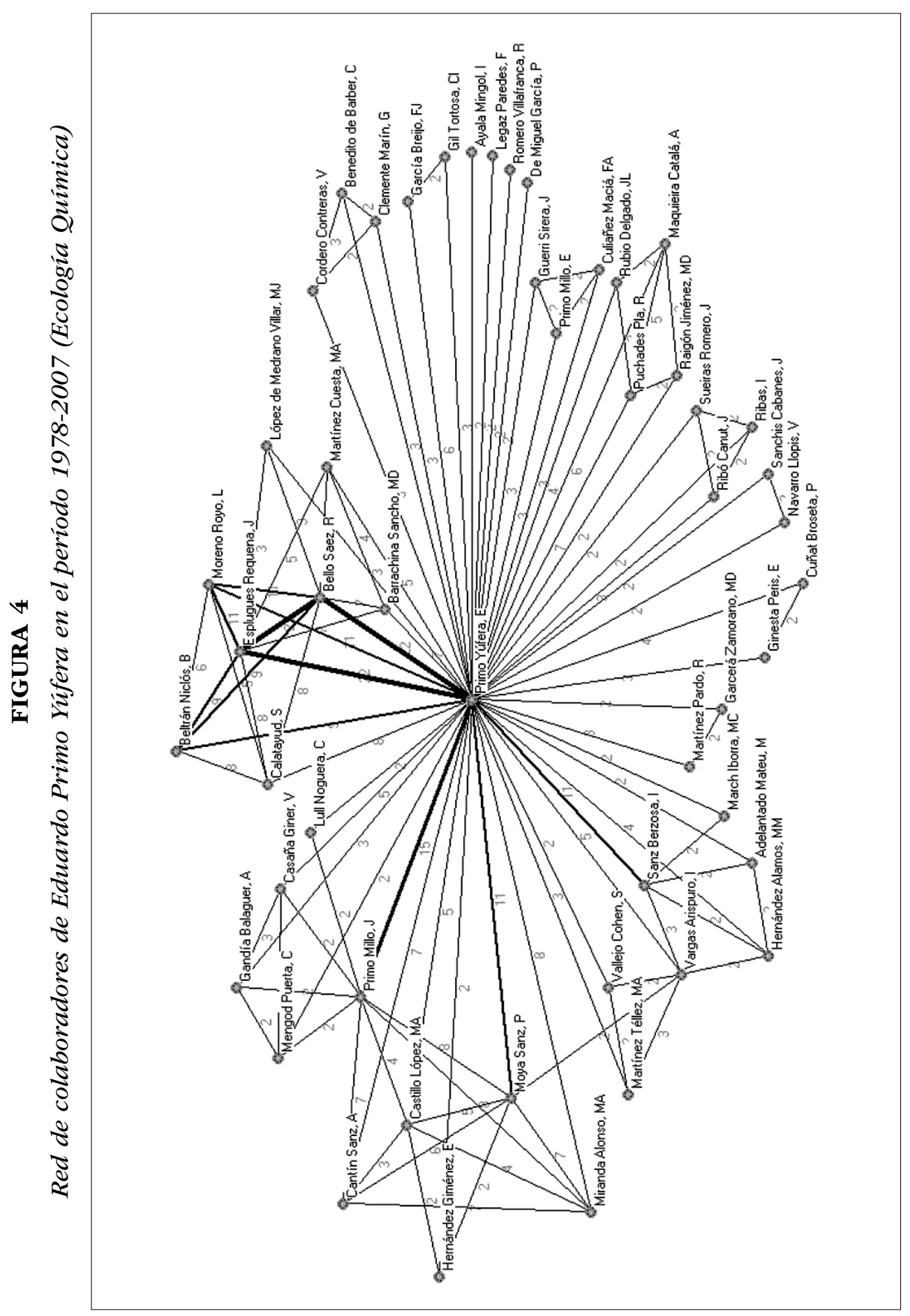




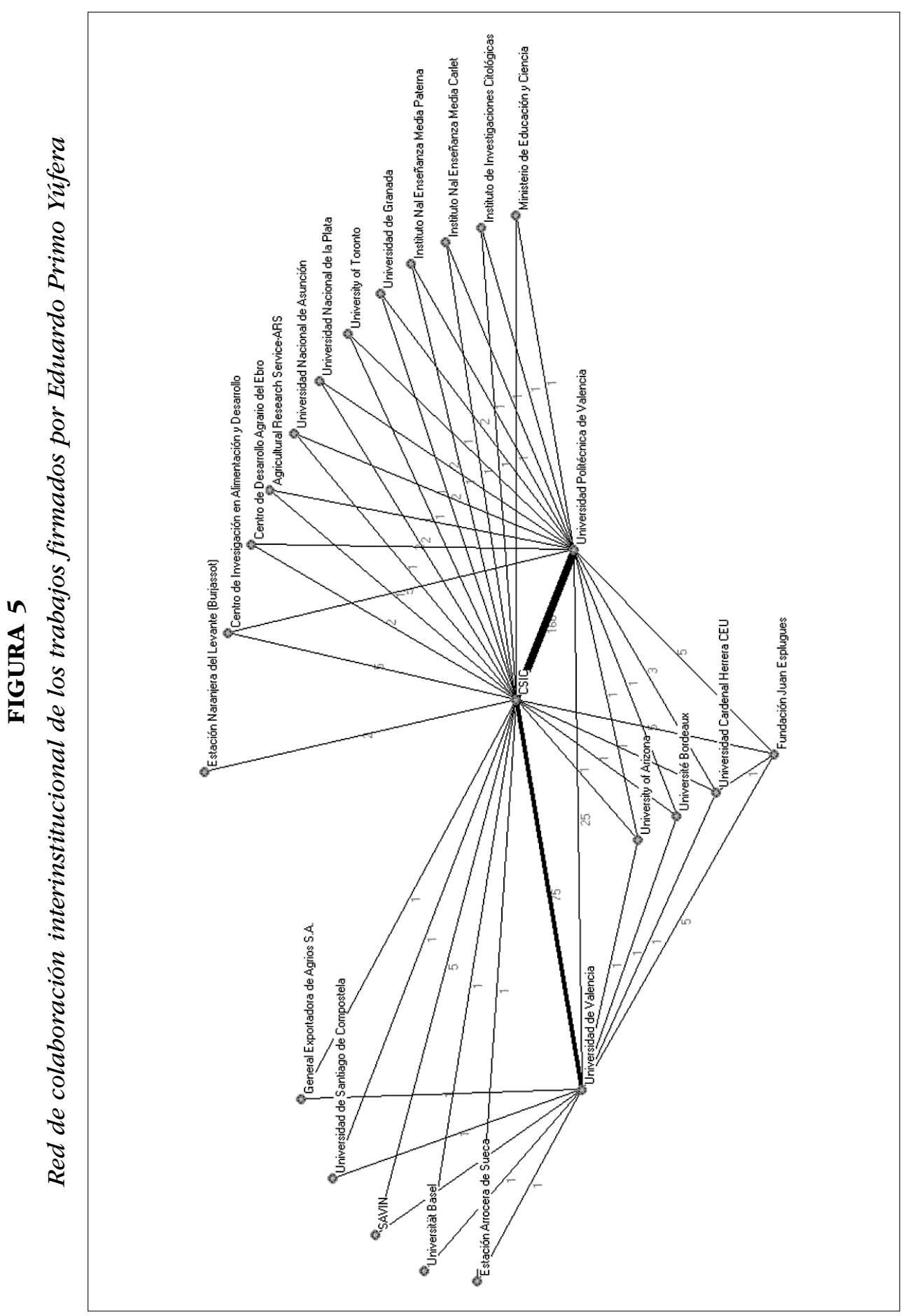


A partir de las firmas institucionales de los artículos publicados por Primo Yúfera y sus colaboradores, se ha realizado la red de las relaciones de colaboración interinstitucional (figura 5). Las relaciones de colaboración institucional más intensas fueron las mantenidas entre el CSIC y la Universidad Politécnica de Valencia, con 166 trabajos en colaboración, el CSIC y la Universidad de Valencia, con 75 trabajos, seguida de la Universidad Politécnica de Valencia y la Universidad de Valencia con 25 trabajos en colaboración. Estas 3 instituciones conforman un triángulo de colaboración de las que derivan el resto de colaboraciones. Todas las instituciones que colaboran lo han hecho con al menos dos de ellas, excepto la Estación Naranjera del Levante, que lo hace sólo con el CSIC. Cinco instituciones colaboraron con el CSIC y la Universidad de Valencia, once instituciones realizaron colaboración con el CSIC y la Universidad Politécnica de Valencia y cuatro instituciones colaboraron con el CSIC, la Universidad Politécnica de Valencia y la Universidad de Valencia.

\subsection{Revistas de publicación, factor de impacto y análisis de citas}

En la tabla VII se recoge la distribución por revistas, países de edición, áreas temáticas a las que se adscriben el conjunto de trabajos publicados, factor de impacto medio y número de citas recibidas. La revista en la que más trabajos publicó Primo Yúfera fue la Revista de Agroquímica y Tecnología de Alimentos (148 trabajos), seguida por Phytotherapy Research (20 trabajos), Anales Real Sociedad Española de Física y Química. Serie B. Química (17 trabajos), Química e Industria (10 trabajos), Revista de Ciencia Aplicada y Farmacognosia: Anales del Instituto José Celestino Mutis (ambas con 8 trabajos), Journal of Agricultural and Food Chemistry y Arbor, Revista de Ciencia, Pensamiento y Cultura (ambas con 7 trabajos). En otras 4 revistas publicó un número de trabajos comprendido entre cinco y tres. El resto de trabajos se dispersan en 45 revistas diferentes, 6 de ellas con dos publicaciones y 39 revistas con un único trabajo. El $50 \%$ de su producción científica quedó recogida en una única revista: la Revista de Agroquímica y Tecnología de Alimentos.

\section{TABLA VII}

Revistas de publicación de los artículos de Eduardo Primo Yúfera

\begin{tabular}{|c|c|c|c|c|}
\hline Revistas & País & Área & $\begin{array}{l}\mathbf{N}^{\mathbf{o}} \text { de } \\
\text { artículos }\end{array}$ & $\begin{array}{c}\text { Factor de } \\
\text { impacto } \\
\text { medio }\end{array}$ \\
\hline $\begin{array}{l}\text { Revista de Agroquímica y Tecno- } \\
\quad \text { logía de Alimentos }\end{array}$ & España & $\begin{array}{l}\text { Ciencia y Tecnología de } \\
\text { Alimentos }\end{array}$ & 148 & 0,132 \\
\hline Phytotherapy Research & Reino Unido & $\begin{array}{l}\text { Química Médica/ Farma- } \\
\text { cología y Farmacia }\end{array}$ & 20 & 0,562 \\
\hline
\end{tabular}


TABLA VII (continuación)

\begin{tabular}{|c|c|c|c|c|}
\hline Revistas & País & Área & $\begin{array}{c}\mathbf{N}^{\mathrm{o}} \text { de } \\
\text { artículos }\end{array}$ & $\begin{array}{c}\text { Factor de } \\
\text { impacto } \\
\text { medio }\end{array}$ \\
\hline $\begin{array}{l}\text { Anales Real Sociedad Española de } \\
\quad \text { Física y Química. Serie B. Quí- } \\
\text { mica }\end{array}$ & España & Química/Física & 17 & - \\
\hline Química e Industria & España & Química & 10 & - \\
\hline $\begin{array}{l}\text { Farmacognosia: Anales del Insti- } \\
\text { tuto José Celestino Mutis }\end{array}$ & España & $\begin{array}{l}\text { Farmacognosia/Farmaco- } \\
\text { logía }\end{array}$ & 8 & - \\
\hline Revista de Ciencia Aplicada & España & $\begin{array}{l}\text { Ciencias Puras.Ciencias } \\
\text { Exactas y Naturales }\end{array}$ & 8 & - \\
\hline $\begin{array}{l}\text { Arbor. Revista de Ciencia, Pensa- } \\
\text { miento y Cultura }\end{array}$ & España & Humanidades & 7 & - \\
\hline $\begin{array}{l}\text { Journal of Agricultural and Food } \\
\text { Chemistry }\end{array}$ & EEUU & $\begin{array}{l}\text { Agricultura/Química Apli- } \\
\text { cada/ Ciencia y Tecno- } \\
\text { logía de Alimentos }\end{array}$ & 7 & 1,53 \\
\hline Revista Las Ciencias & España & Ciencias & 5 & - \\
\hline Plant and Soil & Holanda & $\begin{array}{l}\text { Agronomía/Botánica/ } \\
\text { Edafología }\end{array}$ & 4 & 0,693 \\
\hline $\begin{array}{c}\text { Boletín del Instituto Nacional de } \\
\text { Investigaciones Agronómicas }\end{array}$ & España & $\begin{array}{l}\text { Agricultura/Agronomía/ } \\
\text { Ingeniería Agrícola }\end{array}$ & 3 & \\
\hline Journal of Economic Entomology & EEUU & Entomología & 3 & 0,856 \\
\hline Agrochimica & Italia & Química Aplicada/Botánica & 2 & 0,026 \\
\hline $\begin{array}{l}\text { In: Revista de Información del Ins- } \\
\text { tituto Nacional de Industria }\end{array}$ & España & $\begin{array}{c}\text { Tecnología, Ingeniería e } \\
\text { Industria/Economía y } \\
\text { Política Económica }\end{array}$ & 2 & - \\
\hline $\begin{array}{l}\text { Journal of Applied Entomology- } \\
\text { Zeitschrift Fur Angewandte } \\
\text { Entomologie }\end{array}$ & Alemania & Entomología & 2 & 0,394 \\
\hline Journal of Organic Chemistry & EEUU & Química Orgánica & 2 & 3,488 \\
\hline Pest Management Science & Reino Unido & Agronomía/Entomología & 2 & 1,257 \\
\hline Tecnología Alimentaria & Argentina & $\begin{array}{l}\text { Ciencia y Tecnología de } \\
\text { Alimentos/Industria } \\
\text { Alimentaria }\end{array}$ & 2 & - \\
\hline Agricultural Research & EEUU & Agricultura & 1 & 0,035 \\
\hline $\begin{array}{c}\text { Anales de Química. Serie C. Quí- } \\
\text { mica Orgánica y Bioquímica }\end{array}$ & España & Química & 1 & 0,25 \\
\hline $\begin{array}{l}\text { Anales de Química-International } \\
\text { Edition }\end{array}$ & España & Química & 1 & 0,157 \\
\hline Biological Control & EEUU & $\begin{array}{l}\text { Biotecnología y Microbio- } \\
\text { logía Aplicada/Ento- } \\
\text { mología }\end{array}$ & 1 & 1,299 \\
\hline
\end{tabular}


TABLA VII (continuación)

\begin{tabular}{|c|c|c|c|c|}
\hline Revistas & País & Área & $\begin{array}{c}\mathbf{N}^{\circ} \text { de } \\
\text { artículos }\end{array}$ & $\begin{array}{c}\text { Factor de } \\
\text { impacto } \\
\text { medio }\end{array}$ \\
\hline Biological Wastes & Reino Unido & $\begin{array}{l}\text { Agricultura/ Biotecnología } \\
\text { y Microbiología Apli- } \\
\text { cada }\end{array}$ & 1 & 0,247 \\
\hline Bioresource Technology & Reino Unido & $\begin{array}{l}\text { Ingeniería Agrícola/ Bio- } \\
\text { tecnología y Microbio- } \\
\text { logía Aplicada/Energía } \\
\text { y Combustibles }\end{array}$ & 1 & 0,633 \\
\hline $\begin{array}{l}\text { Bioscience Biotechnology and } \\
\text { Biochemistry }\end{array}$ & Japón & $\begin{array}{l}\text { Bioquímica y Biología Mo- } \\
\text { lecular/ Biotecnología y } \\
\text { Microbiología Aplicada } \\
\text { / Ciencia y Tecnología } \\
\text { de Alimentos }\end{array}$ & 1 & 0,863 \\
\hline $\begin{array}{l}\text { Bulletin of Entomological Re- } \\
\text { search }\end{array}$ & Reino Unido & Entomología & 1 & 1,431 \\
\hline $\begin{array}{l}\text { European Journal of Organic } \\
\text { Chemistry }\end{array}$ & Alemania & Química Orgánica & 1 & 2,15 \\
\hline Grasas y Aceites & España & $\begin{array}{l}\text { Química Aplicada/Ciencia } \\
\text { y Tecnología de Ali- } \\
\text { mentos }\end{array}$ & 1 & 0,44 \\
\hline Helvetica Chimica Acta & Suiza & Química & 1 & 1,692 \\
\hline Journal of Chemical Ecology & Holanda & $\begin{array}{c}\text { Bioquímica y Biología } \\
\text { Molecular/Ecología }\end{array}$ & 1 & 1,237 \\
\hline $\begin{array}{l}\text { Journal of Chromatographic } \\
\text { Science }\end{array}$ & EEUU & $\begin{array}{l}\text { Métodos de Investigación } \\
\text { Bioquímica/Química } \\
\text { Analítica }\end{array}$ & 1 & 1,534 \\
\hline Journal of Horticultural Science & Reino Unido & $\begin{array}{l}\text { Agricultura/Química/Bo- } \\
\text { tánica }\end{array}$ & 1 & 0,553 \\
\hline $\begin{array}{l}\text { Journal of Physiology and Bioche- } \\
\text { mistry }\end{array}$ & España & $\begin{array}{c}\text { Bioquímica y Biología } \\
\text { Molecular/Fisiología }\end{array}$ & 1 & 0,067 \\
\hline Letters in Applied Microbiology & Reino Unido & $\begin{array}{l}\text { Biotecnología y Microbio- } \\
\text { logía Aplicada/Micro- } \\
\text { biología }\end{array}$ & 1 & 1,04 \\
\hline $\begin{array}{l}\text { Methods and Findings in Experi- } \\
\text { mental and Clinical Pharma- } \\
\text { cology }\end{array}$ & España & Farmacología y Farmacia & 1 & 0,237 \\
\hline $\begin{array}{l}\text { Nutrient Cycling in Agroecosys- } \\
\text { tems }\end{array}$ & Holanda & Edafología & 1 & 0,936 \\
\hline Pharmacology and Toxicology & Dinamarca & $\begin{array}{l}\text { Farmacología y Farmacia/ } \\
\text { Toxicología }\end{array}$ & 1 & 1,117 \\
\hline Plant Growth Regulation & Holanda & Botánica & 1 & 0,712 \\
\hline
\end{tabular}


TABLA VII (continuación)

\begin{tabular}{l|l|l|c|c}
\hline \multicolumn{1}{c|}{ Revistas } & \multicolumn{1}{c|}{ País } & \multicolumn{1}{c|}{ Área } & $\begin{array}{c}\mathbf{N}^{\mathbf{d}} \mathbf{\text { de }} \\
\text { artículos }\end{array}$ & $\begin{array}{c}\text { Factor de } \\
\text { impacto } \\
\text { medio }\end{array}$ \\
\hline Planta & Alemania & Botánica & 1 & 2,84 \\
\hline Revista Mexicana de Fitopatología & México & Agronomía & 1 & - \\
\hline Southwestern Entomologist & EEUU & Entomología & 1 & 0,295 \\
\hline Tetrahedron-Asymmetry & Reino Unido & $\begin{array}{l}\text { Química Inorgánica y Nu- } \\
\text { clear /Química Orgáni- } \\
\text { ca/Química-Física }\end{array}$ & 1 & 2,265 \\
\hline Theoretical and Applied Genetics & Alemania & $\begin{array}{l}\text { Agronomía/Botánica/ Ge- } \\
\text { nética y Herencia/ Hor- } \\
\text { ticultura }\end{array}$ & 1 & 1,615 \\
\hline Zentralblatt Fur Mikrobiologie & Alemania & Microbiología & 1 & 0,115 \\
\hline revistas con 1 artículo & EEUU & $\begin{array}{c}\text { Biotecnología y Microbio- } \\
\text { logía Aplicada / Cien- } \\
\text { cia y Tecnología de Ali- } \\
\text { mentos }\end{array}$ & 2 & - \\
\hline 11 revistas con 1 artículo & España & Varias áreas & 11 & - \\
\hline & & Total & 291 & - \\
\hline
\end{tabular}

Primo Yúfera publicó en 36 revistas con factor de impacto entre las que destacan Journal of Organic Chemistry (FI = 3,500 en el año 1998 y FI = 3,476 en el año 1997), seguida de Planta (FI = 2,840 en el año 1982), Tetrabedron-Asymme$\operatorname{try}(\mathrm{FI}=2,265$ en el año 2001), European Journal of Organic Chemistry ( $\mathrm{FI}=2,150$ en el año 2000) y Journal of Agricultural and Food Chemistry (FI = 2,102 en el año 2003). Además, publicó uno o más trabajos en otras 10 revistas con un factor de impacto entre 1 y 2.

El número de citas recibidas, número de trabajos citados, índice citas/trabajo y el factor h (h-index) se han calculado únicamente con los 86 registros recuperados en SCI en marzo de 2011. Estos artículos representan únicamente un 29,55\% de la totalidad de la producción científica objeto de estudio. Han recibido citas el $77,9 \%$ de los trabajos ( $n=64$ ) (tabla VIII).

El $57,8 \%$ de las citas corresponden a quince trabajos (todos han sido citado 12 veces o más). De estos trabajos, el más antiguo es de 1982, diez de ellos pertenecen a la década de los 90 y cuatro a los primeros años del siglo xxi. Catorce trabajos son citados entre 9 y 5 veces, reuniendo el 18,5\% de las citas; veintiún trabajos son citados entre 4 y 2 veces, representando el 12,9\% de las citas totales recibidas por los trabajos, y trece trabajos han sido citados una única vez.

El $86,11 \%$ de las revistas (31 revistas) han recibido citas y de ellas destacan por haber recibido un mayor número de citas, Phytotherapy Research ( $n=81$, el 16,8\% de las citas recibidas), seguida de Journal of Agricultural and Food Che- 
mistry $(n=64)$, Journal of Organic Chemistry $(n=55)$ y Plant and Soil $(n=41)$, que reciben un $13,3 \%$, un $11,4 \%$ y un $8,5 \%$ de las citas, respectivamente. Biological Control y Journal of Economic Entomology reúnen el 13,5\% de las citas ( $n=38$ y $n=27$, respectivamente). Doce revistas reciben entre 8 y 20 citas, y trece revistas entre 1 y 4 citas. El conjunto de trabajos del autor han recibido un total de 482 citas, lo que sitúa la media de citas por trabajo en 7,19.

Los trabajos con más visibilidad internacional vía citas pertenecen a su última etapa como investigador en el área de Ecología Química, cuando ya tenía más de 60 años, en los que el idioma prioritario de publicación fue el inglés (72\%).

El factor h (h-index) del autor es 12; la tabla VIII muestra los 15 artículos que han recibido 12 o más citas y que se han considerado en el cálculo de este indicador.

\section{TABLA VIII}

\section{Hot papers de Eduardo Primo Yúfera}

\begin{tabular}{|c|c|}
\hline Referencia & Citas \\
\hline $\begin{array}{l}\text { Castillo, M. A., Moya, P., Hernández, E., y Primo-Yúfera, E. (2000). Susceptibility of Ce- } \\
\text { ratitis capitata Wiedemann (Diptera: Tephritidae) to entomopathogenic fungi and } \\
\text { their extracts. Biological Control, vol. } 19 \text { (3), 274-282. }\end{array}$ & 38 \\
\hline $\begin{array}{l}\text { Moya, P., Castillo, M. A., Primo-Yúfera, E., Couillaud, F., Martínez, R., Garcerá, M. D., } \\
\text { Miranda, M. A., Primo, J. y Martínez-Pardo, R. (1997). Brevioxime: A new juvenile } \\
\text { hormone biosynthesis inhibitor isolated from Penicillium brevicompactum. Journal } \\
\text { of Organic Chemistry, vol. } 62 \text { (24), 8544-8545. }\end{array}$ & 28 \\
\hline $\begin{array}{l}\text { Legaz, F., Primo, E., Primo-Yúfera, E., Gil, C. y Rubio, L. (1982). Nitrogen fertilization } \\
\text { in citrus. 1. Absorption and distribution of nitrogen in calamondin trees (Citrus mitis } \\
\text { BI), during flowering, fruit set and initial fruit development periods. Plant and Soil, } \\
\text { vol. } 66 \text { (3), 339-351. }\end{array}$ & 28 \\
\hline $\begin{array}{l}\text { Moya, P., Cantín, A., Castillo, M. A., Primo-Millo, J., Miranda, M. A. y Primo-Yúfera, E. } \\
\text { (1998). Isolation, structural assignment, and synthesis of N-(2-methyl-3-oxodecanoyl)- } \\
\text { 2-pyrroline, a new natural product from Penicillium brevicompactum with in vivo } \\
\text { anti-juvenile hormone activity. Journal of Organic Chemistry, vol. } 63 \text { (23), 8530-8535. }\end{array}$ & 27 \\
\hline $\begin{array}{l}\text { Moreno, L., Bello, R., Beltrán, B., Calatayud, S., Primo-Yúfera, E. y Esplugues, J. (1998). } \\
\text { Pharmacological screening of different Juniperus oxycedrus L. extracts. Pharmacolo- } \\
\text { gy and Toxicology, vol. } 82 \text { (2), 108-112. }\end{array}$ & 18 \\
\hline $\begin{array}{l}\text { Casaña, V., Gandía, A., Mengod, C., Primo, J. y Primo-Yúfera, E. (1999). Insect growth } \\
\text { regulators as chemosterilants for Ceratitis capitata (Diptera: Tephritidae). Journal of } \\
\text { Economic Entomology, vol. } 92 \text { (2), 303-308. }\end{array}$ & 17 \\
\hline $\begin{array}{l}\text { González, M. C., Lull, C., Moya, P., Ayala, I., Primo, J. y Primo-Yúfera, E. (2003). Insec- } \\
\text { ticidal activity of penitrems, including penitrem G, a new member of the family } \\
\text { isolated from Penicillium crustosum. Journal of Agricultural and Food Chemistry, vol. } \\
51 \text { (8), 2156-2160. }\end{array}$ & 16 \\
\hline $\begin{array}{l}\text { March C., Sanz I. y Primo-Yúfera E. (1991). Antimicrobial activities on mediterranean } \\
\text { plants. Zentralblatt Fur Mikrobiologie, vol. } 146 \text { (4), 291-295. }\end{array}$ & 15 \\
\hline
\end{tabular}




\section{TABLA VIII (continuación)}

\begin{tabular}{|c|c|}
\hline Referencia & Citas \\
\hline $\begin{array}{l}\text { Navarro, V., Sanchis, J., Ayala, I, Casaña, V. y Primo-Yúfera E. (2004). Efficacy of lufe- } \\
\text { nuron as chemosterilant against Ceratitis capitata in field trials. Pest Management } \\
\text { Science, vol. } 60 \text { (9), 914-920. }\end{array}$ & 14 \\
\hline $\begin{array}{l}\text { Muñoz, J., Corma, A., Primo, J. y Primo-Yúfera E. (2001). Zeolites as pheromone dis- } \\
\text { pensers. Journal of Agricultural and Food Chemistry,vol. } 49 \text { (10), 4801-4807. }\end{array}$ & 14 \\
\hline $\begin{array}{l}\text { Cantín, A., Moya, P., Castillo, M. A., Primo, J., Miranda, M. A. y Primo-Yúfera, E. (1999). } \\
\text { Isolation and synthesis of N-(2-methyl-3-oxodec-8-enoyl)-2-pyrroline and 2-(hept-5- } \\
\text { enyl)-3-methyl-4-oxo-6,7,8,8a-tetrahydro-4H-pyrrolo[2,1-b]-3-oxazine. Two new fungal } \\
\text { metabolites with in vivo anti-juvenile-hormone and insecticidal activity. European } \\
\text { Journal of Organic Chemistry, (1), 221-226. }\end{array}$ & 13 \\
\hline $\begin{array}{l}\text { Hernández, M. M., Sanz, I., Adelantado, M., Ballach, S. y Primo-Yúfera E. (1996). Elec- } \\
\text { troantennogram activity from antennae of Ceratitis capitata (Wied) to fresh orange } \\
\text { airborne volatiles. Journal of Chemical Ecology, vol. } 22 \text { (9), 1607-1619. }\end{array}$ & 13 \\
\hline $\begin{array}{l}\text { Barrachina, M. D., Bello, R., Martínez, M. A., Primo-Yúfera, E. y Esplugues, J. (1997). } \\
\text { Analgesic and central depressor effects of the dichloromethanol extract from Schinus } \\
\text { molle L. Phytotherapy Research, vol. } 11 \text { (4), 317-319. }\end{array}$ & 12 \\
\hline $\begin{array}{l}\text { Bello, R., Barrachina, M. D., Martínez, M. A., Esplugues, J. y Primo-Yúfera, E. (1995). } \\
\text { Evaluation of the acute toxicity, analgesic and CNS activities of different species of } \\
\text { Teucrium genus. Phytotherapy Research, vol. } 9 \text { (4), 277-280. }\end{array}$ & 12 \\
\hline $\begin{array}{l}\text { Ginesta, E., García, F. J. y Primo-Yúfera, E. (1994). Antimicrobial activity of xanthatin } \\
\text { from Xanthium spinosum L. Letters in Applied Microbiology, vol. } 18 \text { (4), 206-208. }\end{array}$ & 12 \\
\hline $\begin{array}{l}\text { Castillo, M. A., Moya, P., Cantín, A., Miranda, M. A., Primo, J., Hernández, E. and Primo- } \\
\text { Yúfera, E. (1999). Insecticidal, anti-juvenile hormone, and fungicidal activities of or- } \\
\text { ganic extracts from different Penicillium species and their isolated active components. } \\
\text { Journal of Agricultural and Food Chemistry, vol. } 47 \text { (5), 2120-2124. }\end{array}$ & 11 \\
\hline $\begin{array}{l}\text { Cantín, A., Moya, P., Miranda, M. A., Primo, J. y Primo-Yúfera, E. (1998). Isolation of } \\
\text { N-(2-methyl-3-oxodecanoyl) pyrrole and N-(2-methyl-3-oxodec-8-enoyl)pyrrole, two } \\
\text { new natural products from Penicillium brevicompactum, and synthesis of analogues } \\
\text { with insecticidal and fungicidal activity. Journal of Agricultural and Food Chemistry, } \\
\text { vol. } 46 \text { (11), 4748-4753. }\end{array}$ & 10 \\
\hline $\begin{array}{l}\text { Aliaga, J. R., Culiañez, F. A., Clemente, G. y Primo-Yúfera, E. (1987). Differentiation of } \\
\text { rice varieties by electrophoresis of embryo protein. Theoretical and Applied Genetics, } \\
\text { vol. } 74 \text { (2), 224-232. }\end{array}$ & 10 \\
\hline $\begin{array}{l}\text { Carrasco, J. M., Cuñat, P., Martínez, M., y Primo-Yúfera, E. (1976). Pesticide residues in } \\
\text { total diet samples, Spain 1971-72. Pesticides Monitoring Journal, vol. } 10 \text { (1), 18-23. }\end{array}$ & 10 \\
\hline 2 trabajos con 9 citas & 18 \\
\hline 2 trabajos con 8 citas & 16 \\
\hline 5 trabajos con 6 citas & 30 \\
\hline 5 trabajos con 5 citas & 25 \\
\hline 8 trabajos con 4 citas & 32 \\
\hline 4 trabajos con 3 citas & 12 \\
\hline
\end{tabular}


TABLA VIII (continuación)

\begin{tabular}{|c|c|}
\hline Referencia & Citas \\
\hline 9 trabajos con 2 citas & 18 \\
\hline 13 trabajos con 1 cita & 13 \\
\hline 19 trabajos sin citas & 0 \\
\hline Total & 482 \\
\hline
\end{tabular}

* Las citas corresponden únicamente a los 86 registros indexados en el Science Citation Index (SCI).

Como se puede observar, el trabajo más citado ha sido "Susceptibility of Ceratitis capitata Wiedemann (Diptera: Tephritidae) to entomopathogenic fungi and their extracts», publicado en el año 2000 en la revista Biological Control, con 38 citas. Dos artículos han recibido 28 citas: uno de ellos publicado en 1997 en la revista Journal of Organic Chemistry, y otro publicado en 1982 en la revista Plant and Soil.

El trabajo más antiguo citado es "Hypoglycemic action of the genus Centaurea" de la revista Farmacognosia: Anales del Instituto José Celestino Mutis, del año 1950 (1 cita).

Las áreas de conocimiento de las revistas que incluyen estos cuatro trabajos con un mayor número de citas son: Biotecnología, Microbiología Aplicada y Entomología (Biological Control), Química Orgánica (Journal of Organic Chemistry) y Agronomía, Botánica y Edafología (Plant and Soil).

Teniendo en cuenta la distribución porcentual de las áreas de publicación de los trabajos, según la clasificación temática de las bases de datos en las que se realizaron las búsquedas, el área de Ciencia y Tecnología de Alimentos se sitúa en primer lugar (41\% de trabajos), seguida por Química (8\%), Farmacología y Farmacia (8\%), Química Médica (5\%), Física (5\%), Agricultura (4\%), Agronomía (3\%), Química Aplicada (3\%) y Entomología (3\%). Las áreas de Ciencias Puras, Ciencias Exactas y Naturales, Farmacognosia, Botánica, Biotecnología, Microbiología Aplicada, Humanidades, Edafología y Ciencias representan, en conjunto, un 14\% de los trabajos. Otras 11 áreas reúnen el 8\% restante de los trabajos.

\section{Conclusiones}

1. La producción científica de Primo Yúfera puede considerarase elevada teniendo en cuenta que publicó 291 artículos a lo largo de su trayectoria investigadora, y que el $70 \%$ de esta producción se produjo en una situación de aislamiento por la dictadura franquista, más extremada si cabe en el capítulo científico, y en el que el reconocimiento científico a través del sistema de citas de las publicaciones no estaba establecido.

2. En su primera etapa científica los ámbitos de investigación destacados fueron la Farmacognosia (1943-1950), y la Ciencia y Tecnología de los 
Alimentos (1951-1977), momento en el que se incorpora al Departamento de Química Vegetal del CSIC, que será la génesis el IATA.

En Farmacognosia destacan los trabajos sobre identificación y extracción de nuevos principios activos de origen natural (alcaloides) y en Ciencia y Tecnología de los Alimentos los estudios sobre las condiciones adecuadas para la liofilización de productos vegetales y de alimentos dedicados a dietas infantiles; investigaciones en la mejora de los procesos de fabricación y optimización de las condiciones de esterilización de las conservas vegetales; definición de parámetros de calidad de los alimentos y de métodos analíticos de control; influencia de los procesos industriales y del almacenamiento sobre la calidad de los alimentos; desarrollo de métodos eficaces para la detección de adulteraciones y fraudes y el análisis de los niveles de contaminación; la enzimología de alimentos, el estudio de las interacciones químicas desfavorables entre proteínas y otros componentes y la influencia de éstas en la calidad organoléptica y en el valor nutritivo.

3. En su segunda etapa científica el ámbito de investigación destacado fue la Ecología Química (1978-2007), con estudios sobre farmacología (aislamiento e identificación de componentes orgánicos de productos naturales con acción insecticida, ovicida, fungicida, bactericida), suelos (propiedades térmicas e influencia del fósforo y del nitrógeno) y obtención de alcohol de biomasa y compuestos con efectos tóxicos para los insectos (métodos ecológicos para la lucha contra plagas mediterráneas basados en hongos entomopatógenos, feromonas y compuestos fototóxicos). Se han traducido en la obtención de 7 patentes, 6 de las cuales han sido licenciadas por diferentes empresas nacionales e internacionales.

4. La proyección de su investigación ha sido preeminentemente a escala nacional, ya que su gran inquietud por el entorno más inmediato le llevó a desarrollar estudios de ámbito regional agrícola difundiendo los resultados de sus investigaciones a través de la Revista de Agroquímica y Tecnología de Alimentos que él había fundado, en la que quedó recogida el $50 \%$ de su producción científica. Por este motivo, el idioma mayoritario de publicación fue el español. Si bien la revista tuvo escasa visibilidad internacional a través de citas, no significa que sus investigaciones no tuviesen una amplia proyección internacional ya que, a través del IATA, se establecieron relaciones y convenios de colaboración con varios países de Hipanoamérica, EEUU y China.

5. Los trabajos con más visibilidad internacional vía citas pertenecen a su última etapa como investigador en el área de Ecología Química, que inició tras abandonar la presidencia del CSIC, cuando ya tenía 60 años y manteniendo esta actividad hasta los 89 años. El idioma prioritario de publicación en esta área fue el inglés (72\%).

6. Publicó en revistas editadas en Alemania, Dinamarca, Holanda, Italia, Reino Unido, Suiza, EEUU, Argentina y México. Y realizó publicaciones 
conjuntas con Universidades o Centros de Investigación de Suiza, México, Francia, Argentina, Paraguay, Canadá y EEUU.

Sus numerosas investigaciones en el ámbito de la Ciencia y Tecnología de Alimentos, la creación del IATA, la fundación de la primera Escuela de Especialización en Ciencia y Tecnología de los Alimentos, cuyos cursos derivaron posteriormente en el primer Máster en Ciencia y Tecnología de Alimentos, su implicación en la creación, en la gestión y en la docencia de la Escuela de Ingenieros Agrónomos de la UPV, ser presidente del Instituto Nacional de Ciencia y Tecnología de Alimentos y vicepresidente de la International Union of Food Science and Technology (IUFOST), le han otorgado el reconocimiento de impulsor de la Ciencia y Tecnología de Alimentos en España.

Este trabajo ha aportado un perfil bibliométrico de Primo Yúfera, en el que se ha cuantificado su aportación a la ciencia valenciana, española y mundial. Los indicadores obtenidos permiten concluir que Primo Yúfera ha sido un profesor e investigador de gran relieve y peso científico reconocidos, relacionado con una extensa red de colaboradores. La información obtenida posee gran valor y significación, pues contribuye a situar su obra en el contexto social e histórico que le corresponde.

\section{Bibliografía}

Aleixandre-Benavent, R.; Ayala-Gascón, M.; Gandía-Balaguer, A.; Moreno-Gálvez, A.; Navarro-Moreno, M. A.; Planes-Ferrer, D. (2011). Eduardo Primo Yúfera. Un adalid de la ciencia. Vida y producción científica. Valencia; Universidad Politécnica de Valencia y Universidad Católica de Valencia.

Aleixandre-Benavent, R.; Valderrama-Zurián, J. C.; Gonzalez-Alcaide, G. (2007). El factor de impacto de las revistas científicas: limitaciones e indicadores alternativos. El profesional de la información, vol. 16 (1), 4-11.

Aleixandre-Benavent, R.; González-Alcaide, G.; Alonso-Arroyo, A.; Bolanos-Pizarro, M.; Castello-Cogollos, L.; Valderrama-Zurián, J. C. (2008). Redes de coautoría y colaboración institucional en Farmacia Hospitalaria. Farmacia Hospitalaria, vol. 32 (4), 226-233.

Aupí, V.; Brines, R. (1994). Eduardo Primo Yúfera. La investigación al servicio de la bumanidad. Valencia; Universidad Politécnica de Valencia. Serie: Homenajes 2.

Ayala-Gascón, M.; Aleixandre-Benavent, R.; Gandía-Balaguer, A. (2011). Eduardo Primo Yúfera, founder of Revista de Agroquímica y Tecnología de Alimentos and pioneer on Food Science and Technology research in Spain. Food Science and Technology International, vol. 17 (6), 549-556.

Batagelj, V.; Mrvar, A. (2001). Pajek [Versión 1.27]. Program for Large Network Analysis. Sloveia; University of Ljubljana.

Borgatti, S. P.; Everett, M. G.; Freeman, L. C. (2002). Ucinet for Windows: Software for Social Network Analysis. Harvard, MA; Analytic Technologies.

Cardona, M. (2003). William Paul: a scientist, a teacher, and a friend. Physica Status Solidi (b), vol. 235 (2), 211-220. 
Castro, E.; Fernández, I. (2007). Iniciativas institucionales e individuales en la creación de estructuras científicas: El Instituto de Tecnología Química. Arbor. Ciencia, Pensamiento y Cultura, vol. 183 (727), 803-819.

Conejero, V.; Beltrán, J. P.; Juliá, J.; Pascual-Leone, A. M. (2008). Necrológica:Sesión Necrológica en Homenaje al Excmo. Sr. Don Eduardo Primo Yúfera. Anales de la Real Academia de Farmacia 74 (4).

González-Alcaide, G.; Agulló-Calatayud, V.; Valderrama-Zurián, J. C.; Aleixandre-Benavent, R. (2009). Participación de la mujer y redes de coautoría en las revistas españolas de Sociología. Revista Española de Investigaciones Sociológicas, vol. 12, 153-166.

González-Alcaide, G.; Alonso-Arroyo, A.; González, J.; Pérez-Sempere, A.; ValderramaZurián, J. C.; Aleixandre-Benavent, R. (2008a). Redes de coautoría y colaboración institucional en Revista de Neurología. Revista de Neurología, vol. 46 (11), 642-651.

González-Alcaide, G.; Valderrama-Zurián, J. C.; Aleixandre-Benavent, R. (2008b). Research fronts and collaboration patterns in Reproductive Biology. Coauthorship networks and institutional collaboration. Fertil Steril, vol. 90(4), 941-56.

González-Alcaide, G.; Valderrama-Zurián, J. C.; Aleixandre-Benavent, R.; Alonso-Arroyo, A.; Granda-Orive J. I.; Villanueva-Serrano, S. (2006). Redes de coautoría y colaboración de las instituciones españolas en la producción científica sobre drogodependencias en biomedicina 1999-2004. Trastornos Adictivos, vol. 8, 78-114.

Hirsch, J. E. (2005). An index to quantify an individual's scientific research output. Proceedings of the National Academy of Science, 102, 16569-16572.

Kademani, B. S.; Kalyane, V. L.; Jange, S. (1999). Scientometric portrait of Nobel laureate Dorothy Crowfoot Hodgkin. Scientometrics, vol. 45 (2), 233-250.

Kademani, B. S.; Kalyane, V. L.; Kumar, V. (2001). Scientometric portrait of Nobel laureate Ahmed Hassan Zewail. Malaysian Journal of Library and Information Science, vol. 6 (2), 53-70.

Kademani, B. S.; Kalyane, V. L.; Kumarv, V. (2002). Scientometric portrait of Nobel laureate Harold W. Kroto. SRELS Journal of Information Management, vol. 39 (4), 409-434.

Kalyane, V. L.; Kademani, B. S. (1997). Scientometric portrait of Barbara McClintock: the Nobel laureate in physiology. KELPRO Bulletin, vol. 1 (1), 3-14.

Kalyane, V. L. y Sen, B. K. (1996). Scientometric portrait of Nobel laureate Pierre-Gilles de Gennes. Malaysian Journal of Library and Information Science, vol. 1 (2), 13-26.

Kalyane, V. L.; Sen, B. K. (2003). Research Productivity of Tibor Braun: An Analytical Chemist-cum-Scientometrician. Annals of Library and Information Studies, vol. 50 (2), 47-61.

López-Piñero, J. M.; Terrada, M. L.; Aleixandre, R.; Valderrama, J. C.; González, G.; Navarro, C. (2008). Santiago Grisolía. Recuerdos. Valencia; Consell Valencià de Cultura.

Maltrás, B. (2003). Los indicadores bibliométricos. Fundamentos y análisis de la ciencia. Gijón; Trea.

Mulla, K. R.; Chandrashekara, M.; Sangam, S. L.; Ramesh, B. B.; Jahagirdar, I. H.; Chowdappa, N.; Nagesh, M. H. (2010). A study on biobibliometric analysis of Prof. C. N. R. Rao's research publications. Sixth International Conference on Webometrics, Informetrics and Scientometrics and Eleventh COLLNET Meeting; University of Mysore.

Newman, M. E. J. (2001). The structure of scientific collaboration networks. Proceedings of the National Academy of Science, vol. 98, 404-409. 
Newman, M. E. J. (2004). Coauthorship networks and patterns of scientific collaboration. Proceedings of the National Academy of Science, vol. 101, 5200-5205.

Peters, H. P. F.; Van Raan, A. F. J. (1994). A bibliometric profile of top-scientists a case study in chemical engineering. Scientometrics, vol. 29 (1), 115-136.

Sangam, S. L.; Savanur, K. P. (2006). Dr. N. Rudraiah: a biobibliometric study. SRELS Journal of Information Management, vol. 43 (2), 185-199.

Sangam, S. L.; Savanur, K. P.; Manjunath, M.; Vasudevan, R. (2006). Scientometric portrait of Prof. Peter John Wyllie. Scientometrics, vol. 66 (1), 43-53.

Sen, B. K.: Gan, S. K. (1990). Bibliometric: Concepts and application in the study of productivity of Scientists. International Forum on Information and Documentation, vol. 15 (3), 13-21.

Subramanyam, K. (1983). Bibliometric studies of research collaboration: A review. Journal of Information, vol. 6 (1): 33-38.

Valderrama-Zurián, J. C.; González-Alcaide, G.; Valderrama-Zurián, F. J.; AleixandreBenavent, R.; Miguel-Dasit, A. (2007). Redes de coautorías y colaboración institucional. Revista Española de Cardiología, vol. 60, 117-130.

Varaprasad, S. J. D.; Sahoo, S.; Madhusudhan, S. (2010). Research contributions of J. S. Yadav to chemical sciences: a scientometric study. Malaysian Journal of Library and Information Science, vol. 15 (2), 41-55. 